\title{
Discriminated conditioned suppression: Further effects of stimulus compounding
}

HOWARD D. CAPPELL, BARBARA HERRING, and C. D. WEBSTER, Alcoholism and Drug Addiction Research Foundation, Toronto 4, Ont., Canada

Rats were given discriminated conditioned-suppression training. After the discrimination was well established, further trials were administered during which the signal for nonshock (CS-) was compounded with the signal for shock (CSt) during selected portions of CS+ presentation. Shocks continued to be delivered following CSt. It was shown that CS- reduced suppression in a readily controllable manner.

With the work of Solomon and his associates as a stimulus (Rescorla \& LoLordo, 1965; Rescorla \& Solomon, 1967), a number of investigators have used operant response rate to assess various aspects of Pavlovian conditioning techniques. Hammond (1966) studied the effects on response rate of pairing one stimulus with shock (CSt) and another with nonshock (CS-) in the Estes-Skinner conditioned-suppression situation. Differential conditioning was readily obtained. Suppression was observed with CS+ but not with CS-; in fact, during early training sessions, $\mathrm{CS}-$ presentations were associated with an acceleration of response rate over the pre-CS level. In a later experiment, Hammond (1967) showed that compounding $\mathrm{CS}-$ with $\mathrm{CS}+$ speeded the extinction of suppression to CS+. The Pavlovian constructs of conditioned inhibition and excitation were used in accounting for Hammond's results. Reberg \& Black (1969) have recently confirmed Hammond's (1967) extinction findings. Additionally, Reberg \& Black (1969) and Miller (1969) have reported that the compounding of two distinct CSts results in greater suppression than either CS+ alone.

The present experiment was designed to provide a further demonstration of the sensitivity of the conditioned-suppression technique in the study of stimulus compounding effects. Two major features of the experiment were that (1) CS+ and $\mathrm{CS}$ - were compounded during acquisition

Fig. 1. Suppression ratios for light CS+ (upper) and tone CS+ (lower) conditionings over the dummy (D), two pretest $(\mathrm{P})$, and the first $\mathbf{1 0}$ conditioning (C) sessions. rather than during extinction and (2) $\mathrm{CS}$ was compounded with CSt only during selected portions of presentations of the latter.

\section{SUBJECTS}

The Ss were four male hooded rats maintained at approximately $75 \%$ of their initial weight ( 90 days old) throughout the experiment.

\section{APPARATUS}

The apparatus consisted of a standard $\mathrm{t} w$ o- b a $\mathrm{r}$ G a son-S ad le r operant-conditioning chamber (Model E3125B-100) with the right-hand bar removed. The chamber, housed in a Grason-Stadler sound-reducing box (Model E3125AA-3), was located in a closet in the experimental room. Two stimuli served as CSs: a tone, intensity $65 \mathrm{~dB}$, frequency $3,000 \mathrm{~Hz}$ (Foringer multiple stimulus panel
Model 1164-4-M1) and two 117-V 7-W lights, one red, one white, which were off for $30 \mathrm{msec}$ of each second. Scrambled shock could be delivered through the grid floor by means of Foringer generator (Model 1154AC) and scrambler (Model 1925) units. Except for Rat C21 during the later part of the stimulus compounding phase, shock intensity was set at 50 on the rat range of the Foringer scale $(1.1 \mathrm{~mA}$ with $100 \mathrm{~K}$ in series with the meter). Shock duration was $0.5 \mathrm{sec}$ throughout. Responses were collected by printout (Grason-Stadler Model 1238).

\section{PROCEDURE}

Acquisition Phase

After shaping, each rat was given four daily 90-min sessions of bar-press training on a VI 60-sec schedule. A reinforcement was a single $45-\mathrm{mg}$ Noyes pellet. The last of these days was a "dummy" day in that records were taken as though the CSs were being presented although these stimuli were actually not in effect.

Two pretest days followed in which the animals were exposed three times to light

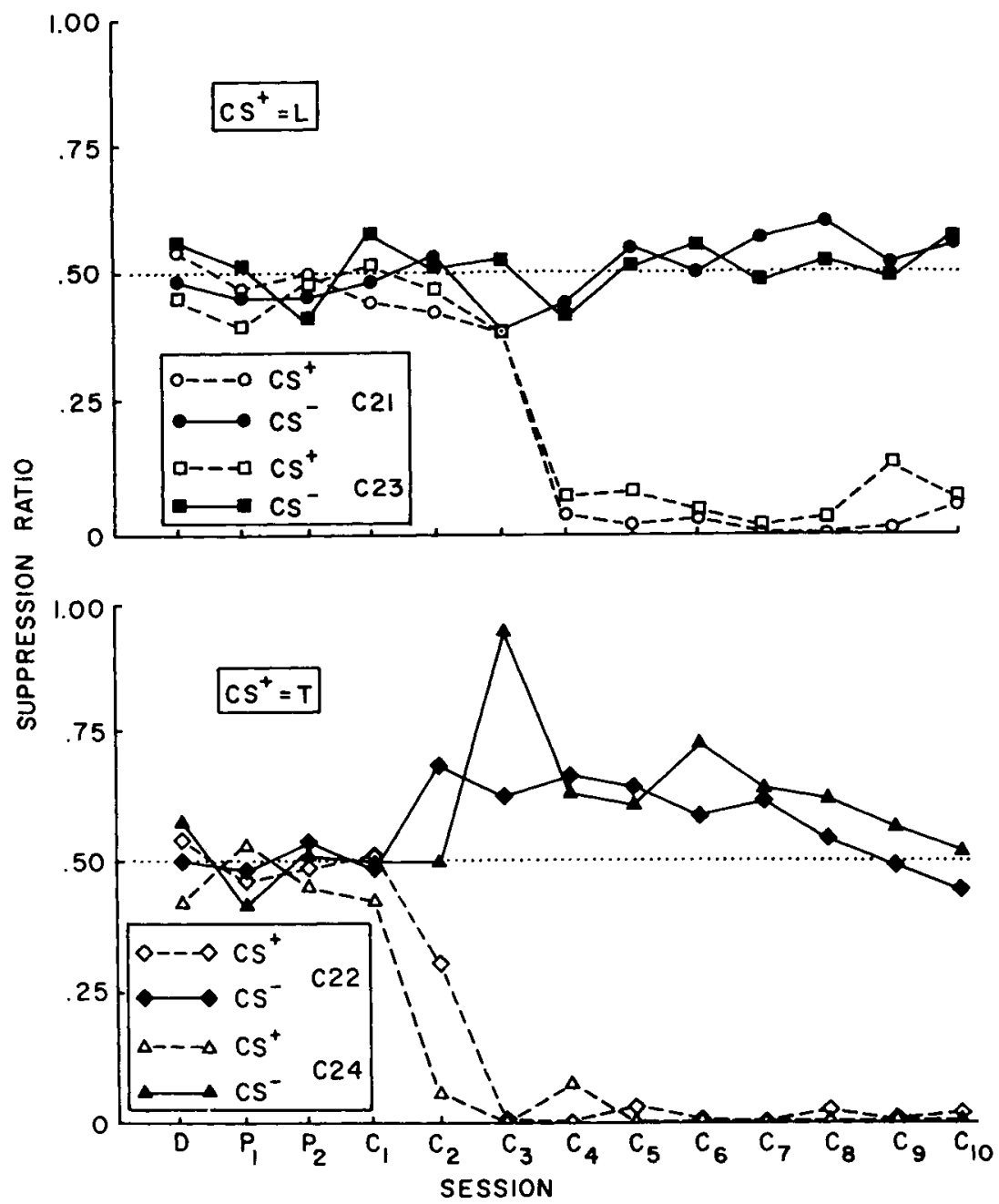


and three times to tone, a single exposure being $3 \mathrm{~min}$ in length. The light and tone were always separated by at least $6 \mathrm{~min}$, and the order of presentation was varied from day to day. Conditioning took place during the next 14 days. Rats C21 and C23 (light $\mathrm{CS}+$ ) received a shock during the last 0.5 sec of the light presentation; Rats C22 and C24 (tone CS+) received an equivalent shock during the last $0.5 \mathrm{sec}$ of each tone presentation. During this phase, the animals were run daily.

\section{Compound Testing Phase}

After acquisition, the animals were given 30 days of compound testing. As during the previous conditioning phase, the CSt and the $\mathrm{CS}-$ were each presented three times for $3 \mathrm{~min}$ during the course of a 90-min session. During each 3-min CS+ presentation, the $\mathrm{CS}$ - was also present for the first or the second minute. This method was designed to show whether or not a compounding effect could be demonstrated within a single presentation of the CS+. The CS- was never presented during the third minute in order to reduce the chance of its becoming a CS+. Whether the $\mathrm{CS}$ - was present in the first or second minute was varied from day to day but was the same for all Ss on a given day. In each block of 10 days, each animal received $\mathrm{CS}$ - during the first minute of the CS+ on 5 days and during the second minute on the other 5. During compound testing, the animals were run daily except on weekends. Shock intensity for one S (C21) was reduced (to a setting of $20,0.4 \mathrm{~mA}$ with $100 \mathrm{~K} \mathrm{ohm}$ in series with the meter) beginning on the 11 th day of the stimulus compounding phase.

\section{RESULTS AND DISCUSSION}

Figure 1 shows the suppression ratios (calculated as $\mathbf{A} / \mathbf{A}+\mathbf{B}$, where $\mathbf{A}$ is the rate during the 3-min CS period and B is the rate during the 3-min pre-CS period) for each $S$ averaged over trials of the dummy, pretest, and differential conditioned-suppression sessions. All four rats clearly showed differential conditioned suppression. However, the phenomenon of increased responding to $\mathrm{CS}-$ during differential conditioning (Hammond, 1966) was more strongly evident when CS - was light and CS+ was tone than when CS- was tone and CS+ was light. Tone was not only relatively ineffective as an inhibitor of suppression when it was CS-;

Fig. 2. Per cent of total responses during each minute of the 3-min CS+ signal-shock interval. Based on the pooled data from the last 10 stimulus compounding sessions. Hatched areas show the minute during which CS- was present. For each S, the histograms on the left are based on five sessions, as are those on the right. it seemed also to have greater effectiveness as a suppressive $\mathrm{CS}+$ than did light. Maximal suppression by tone $\mathrm{CS}+$ was evident by Session C3, while in the case of light CSt maximal suppression did not appear until Session $C 4$.

The effects of compounding CS+ and $\mathrm{CS}-$ are shown in Fig. 2, which gives for each $S$ the percentage 1 response output during presentations of $\mathrm{CS}+$ alone and $\mathrm{CS}+$ compounded with CS-. The data in Fig. 2 are taken from the last 10 compounding sessions (five with $\mathrm{CS}-$ in the first minute, five with $\mathrm{CS}-$ in the second minute) and are based on averages over three trials per session. Similar results were observed throughout the previous compounding sessions, except in the case of Rat C21. It was only after the extremely powerful suppressive effect of CS+ was weakened for Rat $\mathrm{C} 21$ by decreasing shock intensity that CS- effects during compounding could be observed.

From Fig. 2, it can be seen that, although evidence of facilitation of responding by $\mathrm{CS}$ - during differential conditioning was no longer apparent during later acquisition sessions (Fig. 1), stimulus compounding revealed that $\mathrm{CS}-$ had not lost its ability to exert control over response rate. Once more, however, CSwas more effective in controlling behavior when it was light than when it was tone. For Rats C22 and C24, the inhibition of suppression was "turned on" during CSpresentation and abruptly "turned off" when CS - was terminated. In the case of tone CS-, this "on and off" effect was not shown as clearly, though during Minute 1

$$
C s^{+}=L
$$
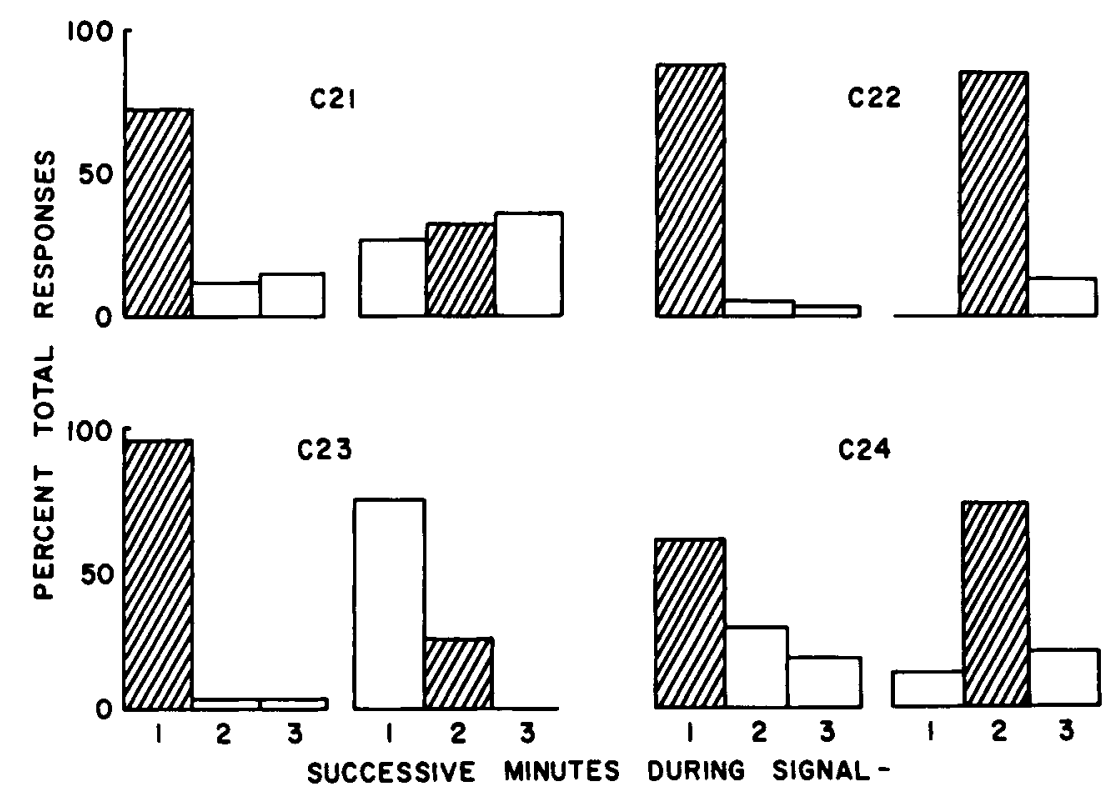

of the signal-shock interval tone did have strong inhibitory effects on conditioned suppression, which terminated abruptly with $\mathrm{CS}-$ offset. Though tone $\mathrm{CS}-$ tended to have weaker countersuppressive effects when presented during Minute 2, response rate during this portion of the signal-shock interval was nonetheless higher when CSwas present than when it was absent.

of some interest was a transitory phenomenon associated with the 3-min presentations of CS- by itself during early compounding sessions. Again, the phenomenon appeared only when CS- was light. For Rat $\mathrm{C} 22$, suppression ratios to $\mathrm{CS}-$ (averaged over the three presentations of each session) were $.56, .63$, and .55 for compounding Sessions $\mathrm{C} 1, \mathrm{C} 2$, and $\mathrm{C} 3$, respectively. These ratios exceeded the suppression ratios to $\mathrm{CS}-$ of the last seven differential conditioning sessions prior to the introduction of stimulus compounding. In the case of Rat $\mathrm{C} 24, \mathrm{CS}$ - suppression ratios of $.58, .60$, and .56 on compounding Sessions 2, 3, and 4 exceeded (or for one session, equaled) the suppression ratios to CS- of the last six differential conditioning sessions prior to compounding. Thus, an explicit pairing of CS- with CS+ temporarily restored suppression ratios to $\mathrm{CS}$ - to the high levels observed early during acquisition of the discrimination.

In summary, though inhibition of conditioned-suppression as a consequence of stimulus compounding has been shown during extinction (Hammond, 1967; Reberg \& Black, 1969), the present experiment is novel in demonstrating

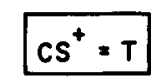

C24

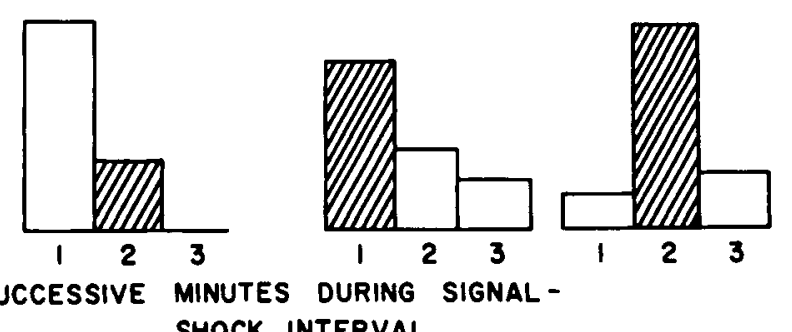


inhibitory stimulus control by $\mathrm{CS}-$, which could be "turned on and off" during consecutive minutes of the same conditioning trial.

\section{REFERENCES}

HAMMOND, L. J. Increased responding to $\mathrm{CS}^{-}$ in differential CER. Psychonomic Science, 1966, 51, 337-338.

HAMMOND, L. J. A traditional demonstration of the active properties of Pavlovian inhibition using differential CER. Psychonomic Science, 1967, 9, 65-66.

MILLER, L. Compounding of pre-aversive stimuli. Journal of the Experimental Analysis of Behavior, 1969, 12, 293-299.

REBERG, D., \& BLACK, A. H. Compound testing of individually conditioned stimuli as an index of excitatory and inhibitory properties. Psychonomic Science, 1969, 17 30-31.

RESCORLA, R. A., \& LOLORDO, V. M. Inhibition of avoidance behavior. Journal of Comparative \& Physiological Psychology, $1965,59,406-412$.

RESCORLA, R. A., \& SOLOMON, R. L. Two-process learning theory: Relationships between Pavlovian conditioning and instrumental learning. Psychological Review, $1965,74,151-182$.

\section{NOTES}

1. The total number of responses made when CS- appeared in Minute 1 were 33,128, 29, and 240 for Rats C21, C22, C23, and C24, respectively; when CS- appeared in Minute 2, the corresponding response totals were 69,41 , 34 , and 120.

\section{Effect of competition on escape from noxious stimulation}

LOUIS W. SUTKER, LAWRENCE GUBLER, JR., and C. J. WALLACE, Loyola University of the South, New Orleans, La. 70118

Twenty 90-day-old male Sprague-Dawley rats were separated into five homogeneous groups of four rats each on the basis of running speed to escape electrical shock in a runway. Within each of these groups, two $S s$ were assigned randomly to a competition condition and two to a noncompetition condition. All Ss were run in pairs in a double-alley runway under shock-escape conditions. The first animal in the competition pair to reach the goal was allowed to enter the goal and escape the shock. Ss in the noncompetition pairs were yoked to the competition pairs, and their reinforcement was contingent upon the performance of their yoked partners. In all cases, the performance of the competition Ss exceeded that of the noncompetition $S s \quad(p<.01)$, demonstrating the motivating effect of stimuli associated with the competitive situation.

Bayroff (1940) has demonstrated a competitive effect (increase in performance due to being allowed reinforcement in the presence of a nonreinforced $S$ ) in rats swimming in an underwater maze. The first rat to reach the goalbox was rewarded by immediate access to air, while the losing rat was deprived for an additional $20 \mathrm{sec}$. Of the total of $56 \mathrm{Ss}$ (28 pairs) $80.4 \%$ swam significantly faster under competition than under noncompetitive conditions.

Church (1962) has pointed out the methodological deficiencies of Bayroff's (1940) study. He has suggested that the latter's results were based on experimentation in which there was no control group to determine whether the increase in performance was due to such factors as (1) the additional number of trials, (2) the delay of reinforcement, or (3) the competitive allotment of reinforcement. Taking these factors into consideration, Church (1962) studied the effect of a competitive situation on lever pressing by albino rats in adjacent cages. On a 30-sec VI schedule, a food reinforcement was made available to both rats, and the first of the two experimental Ss to make the appropriate response received the pellet. These two experimental Ss were matched to a control pair rewarded not on the basis of their own response, but that of the experimental pair. Ss in the former group displayed a considerably greater increase in response rate over those in the control, noncompetition group.

Kanak \& Davenport (1967) used the design of Church (1962) and modified it slightly in order that the motivational influences of the $S s$ were more readily observable. By using the yoked design, competition effects on albino rats in a double-alley runway were studied. Sixteen male albino rats were divided into four homogeneous blocks by rank ordering the mean speed scores of five trials on the 3rd day of training. In each group, one pair was assigned randomly as competitive and the other as noncompetitive. Reinforcement in the competition pair was based on arriving at the goalbox first and being allowed entrance to the food pellets within. In the noncompetition pair, reinforcement was based not on their performance in the runway, but upon that of their yoked competition partner. The results were significant at the .05 level, demonstrating a competition effect.

The present study employed the noxious stimulation of Bayroff's (1940) investigation with the methods and design of Church (1962) and Kanak \& Davenport (1967) to explore the competition effect in the albino rat in escaping electric shock in a double-alley runway.

\section{APPARATUS}

The apparatus consisted of a straight double-alley runway, 48 in. in length, with 8-in.-long start- and goalboxes making the total length $64 \mathrm{in}$. Total width of the runway was $12 \mathrm{in}$., $6 \mathrm{in}$. for each half. The runway was $8 \mathrm{in}$. high and was provided with a clear plastic cover. The doors of the start- and goalboxes and the partition between the two alleys were made of clear Plexiglas. The doors to the start- and goalboxes were of the guillotine type raised by means of a string attached to the top of the Plexiglas and run through a support immediately above. Four microswitches, attached individually to the doors, operated timers to record latency and running speed. The startbox and alley had a grid floor connected to a Grason-Stadler electromechanical scrambling mechanism that provided the noxious stimulation. Shock intensity was $.8 \mathrm{~mA}$. The entire runway, except for the Plexiglas portions and the grid floor, was constructed of $1 / 4$-in. plywood sprayed on the inside with flat black paint.

SUBJECTS AND PROCEDURE

The Ss were 20 male Sprague-Dawley rats, approximately 90 days old, housed in individual cages with ad lib food and water supply. All Ss were rank-ordered on the basis of running speed following a 2-day initial escape training period of 30 trials per day on $100 \%$ reinforcement. Five homogeneous groups of four rats each were formed by placing the fastest four Ss together, then the next fastest four, and so on, down to the slowest four Ss. Within each of these blocks, two Ss were assigned randomly to a competition pair and two to a yoked noncompetition pair. The same pairs of rats always ran together.

Reinforcement was defined as entrance into the goalbox, where no shock was present, and was given only to the first rat reaching the goal chamber in the competition pair and to the rat in the noncompetition pair yoked to the winning $S$ regardless of performance. The losing $S$ and his yoked noncompetition partner were unrewarded, i.e., deprived entrance into the goalbox and maintained under shock for an additional $3 \mathrm{sec}$ for that 